$\mathrm{RM}-75-58$

AN OPTIMAL CONTROL APPROACH TO NATIONAL SETTLEMENT SYSTEM PLANNING

R. K. Mehra

November 1975

Research Memoranda are informal publications relating to ongoing or projected areas of research at IIASA. The views expressed are those of the author, and do not necessarily reflect those of IIASA. 


\section{An Optimal Control Approach to}

National Settlement System Planning

R. K. Mehra*

\section{$\underline{\text { Abstract }}$}

In this paper, an optimal control approach to a problem in national settlement system planning is presented. The problem description is the same as considered by Mackinnon [6] and by Evtushenko and Mackinnon [4]. It is shown how the special structure of the model and the singular nature of the control can be used to reduce the solution of a nonlinear programming problem to the solution of sets of linear equations. $A$ branch and bound integer programing algorithm is used to handle inequality constraints on the control variables. The organization of the paper is as follows. Section I considers problem formulation and an optimal control solution is discussed in Section II. A branch and bound technique for determining active constraints is presented in section III. A more general problem is considered in section IV and conclusions are stated in section $\mathrm{V}$.

\section{Problem Formulation}

We consider first the forward linkage model $(1,2)$ in which the state vector $\mathrm{x}(t) \in \mathrm{R}^{\mathrm{n}}$ represents population distribution at time $t$ and the control vector $u(t) \in R^{n}$ represents migrations to each region from outside the system.

$$
x(t+1)=P x(t)+u(t), t=0, \ldots, T-1 .
$$

The budgetary and fixed immigration constraints on $u(t)$ are expressed as

* International Institute for Applied Systems Analysis, Laxenburg, Austria, and Harvard University. 


$$
\begin{aligned}
& \sum_{t=0}^{T-1} u^{T}(t) r(t) \leq b \\
& \sum_{t=1}^{n} u_{i}(t)=\hat{u}(t), \quad t=0, \ldots, T-1 \\
& u(t) \geq 0 .
\end{aligned}
$$

The initial state $x(0)=x_{0}$ is also specified. The objective function to be minimized with respect to $u(t), t=0, \ldots, T-1$ is

$$
J=\frac{1}{2} \sum_{t=1}^{T}(x(t)-g(t)){ }^{T} Q(x(t)-g(t)) \text {. }
$$

$\mathrm{J}$ measures the deviation of actual populations $\mathrm{x}(t)$ from certain desired population levels $g(t)$. The backward linkage model $(1,2)$ will be considered in section IV.

\section{An Optimal Control Solution}

By adjoining the constraints to the cost function using appropriate multipliers, we define the Lagrangian function (3)

$$
\begin{aligned}
\mathscr{L}= & \sum_{t=1}^{T} \frac{1}{2}(x(t)-g(t))^{T} Q(x(t)-g(t)) \\
& +\sum_{t=0}^{T-1}\left\{\lambda^{T}(t+1)[x(t+1)-P x(t)-u(t)]\right. \\
& \left.+v(t)[\pi u(t)-\hat{u}(t)]+n^{T}(t) u(t)\right\} \\
& +\mu\left(\sum_{t=0}^{T-1} r^{T}(t) u(t)-b\right)+\lambda^{T}(0)\left(x(0)-x_{0}\right),
\end{aligned}
$$

where $\pi=[1, \ldots, 1]$ is a $1 \times n$ vector of all ones.

Here $\lambda(t), \nu(t), n(t)$ and $\mu$ are dual or shadow-price variable for constraints (1), (3), (4) and (2), satisfying constraints $\eta(t) \leq 0$ and $\mu \geq 0$ and having the same dimension as the constraining equation.

By rearranging terms, Eq. (6) can be written as, 


$$
\begin{aligned}
\mathscr{L}= & \frac{1}{2}(x(T)-g(T))^{T} Q(x(T)-g(T)) \\
& +\sum_{t=0}^{T-1}\left\{\frac{1}{2}(x(t)-g(t)) T Q(x(t)-g(t))+\lambda^{T}(t) x(t)\right. \\
& -\lambda^{T}(t+1)(P x(t)+u(t))+v(t)\left(\pi^{T} u(t)-\hat{u}(t)\right) \\
& \left.+\eta^{T}(t) u(t)+\mu\left(r^{T}(t) u(t)-\frac{b}{T}\right)\right\} \\
& +\lambda^{T}(T) x(T)-\lambda^{T}(0) x(0)-(x(0)-g(0))^{T} Q(x(0)-g(0)) \\
& +\lambda^{T}(0)\left(x(0)-x_{0}\right) .
\end{aligned}
$$

Since $\mathscr{L}$ is linear in $u(t)$, the optimal control problem is singular in the sense of Bryson [2].

setting

$$
\frac{\partial \mathscr{L}}{\partial \mathrm{x}(\mathrm{t})}=0, \mathrm{t}=0, \ldots, \mathrm{T}
$$

and

$$
\frac{\partial \mathscr{L}}{\partial u(t)}=0, \quad t=0, \ldots, T-1,
$$

we obtain the following $(2 \mathrm{~T}+1) \mathrm{n}$ linear equations:

$$
\begin{aligned}
& \lambda(t)=P^{T} \lambda(t+1)-Q(x(t)-g(t)), t=1, \ldots, T-1 \\
& \lambda(T)=-Q(x(T)-g(T)) \\
& \lambda(0)=P^{T} \lambda(1)
\end{aligned}
$$

and

$$
\lambda(t+1)=\pi^{T} \nu(t)+r(t) \mu+\eta(t)
$$

Supposing that the constraint $u(t) \geq 0$ is active only at $\mathrm{p}$ points, 1 we now present a method for solving Eq. (8) to (11)

$1_{\text {This implies that } u_{j}}\left(t_{i}\right)=0$ for only $p$ different values of $\left(j_{i}, t_{i}\right)$ where $j_{i} \in[1,2, \ldots, n]$ and $t_{i} \in[0, \ldots, T-1]$. We denote the $\left(j_{i}, t_{i}\right)$ set by $I_{p}$. 
along with Eqs. (1), (2), (3) and p equations from (4), a total of $(3 n T+T+n+1+p)$ equations for an equal number of unknowns

$$
\begin{aligned}
\text { viz. } \quad & (x(t), t=1, \ldots, T),(u(t), t=0, \ldots, T-1), \\
& (\lambda(t), t=0, \ldots, T),(v(t), t=0, \ldots, T-1), \\
& \left.\mu,\left(\eta_{j_{i}}\left(t_{i}\right), j_{i} \in[1, n], t_{i} \in[0, T-1]\right)\right\} .
\end{aligned}
$$

We have assumed here that the budget constraint (2) is binding. Otherwise $\mu=0$ and $\mathrm{Eq}$. (2) is irrelevant. A determination of the binding or nonbinding nature of the constraint Eq. (2) can be done by the branch and bound technique to be described below for determining the values of $t_{i}$ and $j_{i}$ for which the nonnegativity constraints (4) are binding.

The linear nature of the above equations allows us to solve for $(3 \mathrm{nT}+\mathrm{n})$ unknowns in terms of the remaining $(\mathrm{T}+\mathrm{p}+1)$ unknowns $\theta$ where $\theta=\left\{v(t), t=0, \ldots, T-1 ; \mu ; \eta_{j_{i}}\left(t_{i}\right)\right.$, $\left.\left(j_{i}, t_{i}\right) \in I_{p}\right\}$. This is a significant reduction in computation since $n$ may be the order of twenty to 100 and $T$ of the order three to ten.

Eq. (11) gives $\lambda(t)$ in terms of $\theta$. Substituting for $\lambda(t)$ in Eqs. ( 8$)$ to $(10)$, we can also obtain $x(t)$ in terms of $\theta$. Then $\mathrm{u}(\mathrm{t})$ can be solved from Eq. (1). Now there are exactly $(\mathrm{T}+\mathrm{p}+1)$ binding constraints on $u(t)$ and these give us the desired equations for $(T+p+1)$ unknowns, $\theta$. The detailed equations are

where

$$
\begin{aligned}
x(t)= & g(t)-Q^{-1} \lambda(t)+Q^{-1} P^{T} \lambda(t+1) \\
= & g(t)-Q^{-1}\left(\pi^{T} v(t-1)+r(t-1) \mu+n(t-1)\right) \\
& +Q^{-1} P^{T}\left(\pi^{T} v(t)+r(t) \mu+n(t)\right)
\end{aligned}
$$

$$
\begin{aligned}
t & =1, \ldots, T-1 \\
x(t) & =g(T)-Q^{-1}\left(\pi^{T} v(T-1)+r(T-1) \mu+\eta(T-1)\right) \\
\lambda(0) & =P^{T}\left[\pi^{T} v(0)+r(0) \mu+\eta(0)\right] .
\end{aligned}
$$


From Eq. (1), for $t=1, \ldots, T-2$

$$
\begin{aligned}
& u(t)=x(t+1)-P x(t) \\
& =g(t+1)-Q^{-1}\left(\pi^{T} \nu(t)+r(t) \mu+n(t)\right) \\
& +Q^{-1} P^{T}\left(\pi^{T} \nu(t+1)+r(t+1) \mu+n(t+1)\right) \\
& -P\left[g(t)-Q^{-1}\left(\pi^{T} v(t-1)+r(t-1) \mu+n(t-1)\right)\right. \\
& \left.+Q^{-1} P^{T}\left(\pi^{T} \nu(t)+r(t) \mu+n(t)\right)\right] \\
& u(0)=g(1)-Q^{-1}\left(\pi^{T} \nu(0)+r(0) \mu+n(0)\right)+Q^{-1} P^{T}\left(\pi^{T} \nu(1)\right. \\
& +r(1) \mu+\eta(1))-\mathrm{Px}_{0} \\
& u(T-1)=g(T)-Q^{-1}\left(\pi^{T} v(T-1)+r(T-1) \mu+\eta(T-1)\right) \\
& -P\left[g(T-1)-Q^{-1}\left(\pi^{T} v(T-2)+r(T-2) \mu\right.\right. \\
& +\eta(T-2))+Q^{-1} P^{T}\left(\pi^{T} \nu(T-1)+r(T-1) \mu\right. \\
& +n(T-1))] \text {. }
\end{aligned}
$$

From Eq. (2), (3) and (4), and assuming $\nu(T)=0, r(T)=\eta(T)=0$, $v(-1)=0, r(-1)=\eta(-1)=0$, an equation of the form $A \theta=c$ is obtained where

$$
\theta=\left[\begin{array}{l}
v(0) \\
\vdots \\
v(T-1) \\
\mu \\
\eta_{j}\left(t_{1}\right) \\
\eta_{j_{k}}\left(t_{k}\right)
\end{array}\right] \text {. }
$$

The times at which the constraints $u(t)=0$ are binding $t_{1} \leq t_{2} \ldots \leq t_{k}$, and $j_{1}, \ldots, j_{i}$ are the corresponding control variables. If two or more control variables are zero at one time, then the time index is repeated. 


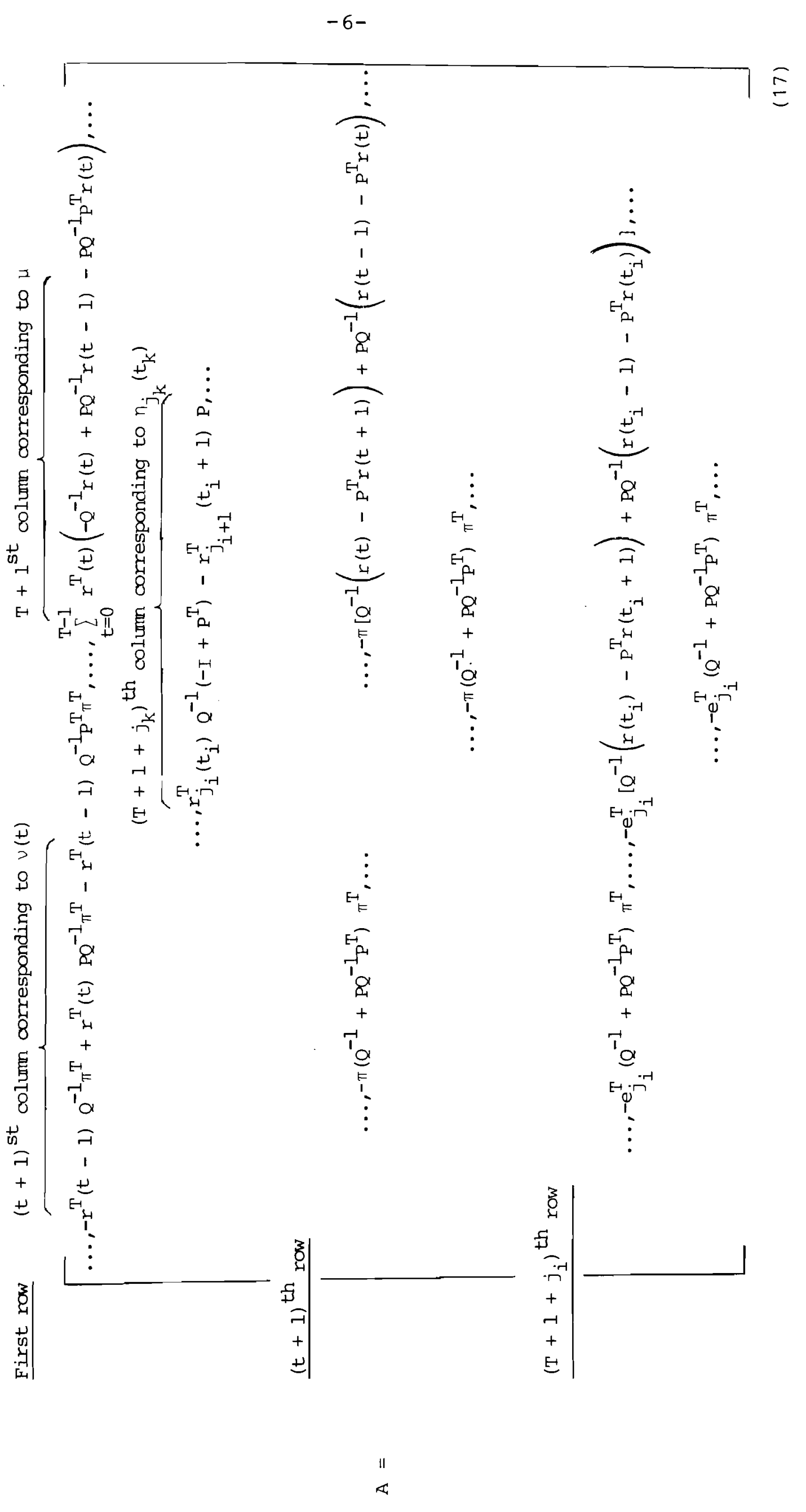




$$
c=\left[\begin{array}{ll}
b & \sum^{T-1} r^{T}(t)\{g(t+1)-P g(t)\} \hat{u}(t) \\
& -\pi g(t+1)+\pi P g(t) \\
& -e_{j_{i}}^{T}\left(g\left(t_{i}+1\right)-P g\left(t_{i}\right)\right)
\end{array}\right]
$$

where $e_{j}$ is an $n \times 1$ vector of all zeros except 1 at location $j_{i}$ Assuming $A$ is nonsingular, we obtain

$$
\theta=A^{-1} \mathrm{C}
$$

If $A$ is singular, but $c$ belongs to the range space of $A$, we can still solve for $\theta$ but it is no longer unique. However, this is an indication that not all the constraints are linearly independent and a reduction in the dimension of $\theta$ can be made. This may be achieved by changing $p$, the number of controls that are exactly zero. If $A$ is singular and $c$ does not belong to the range space of $A$, the constraints are inconsistent and some of the constráints must be dropped. For example, Eq. (3) dictates that not all the controls at a given time can be identically zero. If an attempt to do so is made in Eq. (4), an inconsistent set of equations for $\theta$ will result. We now examine the problem of identifying the controls that are identically zero.

\section{Branch and Bound Integer Programming Technique}

The total number of control variables is $\mathrm{nT}$, but due to constraint (3) at most ( $n-1)$ T control variables can be identically zero. (See Garfinkel and Nemhauser [5].) I denotes the subset of controls that are zero for a particular solution trajectory and let $I^{*}$ denote the zero control subset for the optimal trajectory. We are interested in determining $I^{*}$ by an efficient search over all admissible $I_{p^{\prime}} p=0,1, \ldots,(\mathrm{n}-1) \mathrm{T}$.

A branch and bound technique (see [5]) may be used as follows: ${ }^{2}$

1) Start with $I_{0}$, an empty set, i.e. neglect all nonnegativity constraints. Solve Eq. (19) and determine the corresponding control set, $\mathrm{U}_{0} \cdot$ The associated cost $\mathrm{J}_{0}$ is a lower bound on the optimal cost $\mathrm{J}^{*}$, since the addition of constraints can only increase the cost. Make $U_{0}$ feasible by setting all negative controls to zero and let the corresponding control by $U_{0}^{\prime}$ with

2 The author is grateful to Dr. David E. Bell for discussions on this subject. 
associated cost $\mathrm{J}_{0}^{\prime}$. Then $\mathrm{J}_{0}^{\prime}$ is an upper bound on $\mathrm{J}^{*}$.

$$
\mathrm{J}_{0} \leq \mathrm{J} * \mathrm{~J}_{0}^{\prime}
$$

Store $U_{0}, J_{0}, U_{0}^{\prime}$ and $J_{0}^{\prime}$. Notice that $U_{0}^{\prime}$ is easily constructed from $\mathrm{U}_{0}$ so that it need not be stored if $\mathrm{U}_{0}$ is stored.

2) Now add one element to the set $I_{0}$, i.e. only one control variable is set to zero. Examine all sets $I_{1}$ of a single element and let $I_{1}^{*}$ be the set with the least cost $J_{1}$. Notice that the addition of a single extra constraint increases the dimension of $\theta$ by one and adds a new row and column to $A$. The inversion of the augmented $A$ can be done in terms of the previous A by using the Frobenius relation (5) or the matrix sweep methods (6). Thus no further inversions of matrix $A$ are required as long as the addition or subtraction of constraints is done one at a time. Let $U_{1}$ be the optimal control under $I_{1}^{*}$ and $U_{1}^{\prime}$ be the projected feasible solution with $\operatorname{cost} J_{1}$. Notice that $J_{1}$ will provide an improved lower bound on $J^{*}$, but $J_{1}^{\prime}$ need not be an improved upper bound. At any stage of the iteration, we store the best upper and lower bounds and the associated control histories.

3) We now examine sets $I_{2}$ of two elements, obtained from $I_{1}^{*}$ by adding one more constraint. Let $I_{2}^{*}$ be the set providing the minimal cost $\mathrm{J}_{2}$, control $\mathrm{U}_{2}$, projected feasible control $\mathrm{U}_{2}^{\prime}$ and the corresponding cost $\mathrm{J}_{2}^{\prime} \cdot$ If $\mathrm{J}_{2}$ is less than the best upper bounds, we accept it as the best lower bound since by construction $\mathrm{J}_{2} \geq \mathrm{J}_{1} \geq \mathrm{J}_{0}$. But if $\mathrm{J}_{2}$ exceeds the best upper bound, then $I_{2}^{*}$ and all its predecessors (see Figure 1) are excluded from further consideration. In this case, we go back to a set $I_{1}$ of a single element by eliminating the common variable among $I_{1}^{*}$ and $I_{2}^{*}$ using a matrix sweep algorithm (6). We then go down this branch of the tree in the same fashion till either $U$ and $U$ ' become identical or the lower bound exceeds the best upper bound achieved previously.

The branch and bound procedure may be made more efficient by using information about multipliers $n(t)$. If at any stage of the calculation, $n(t)$ turns out to be zero or positive for a control variable on the constraint boundary, it is an indication that the corresponding control variable should be taken off the constraint boundary. 


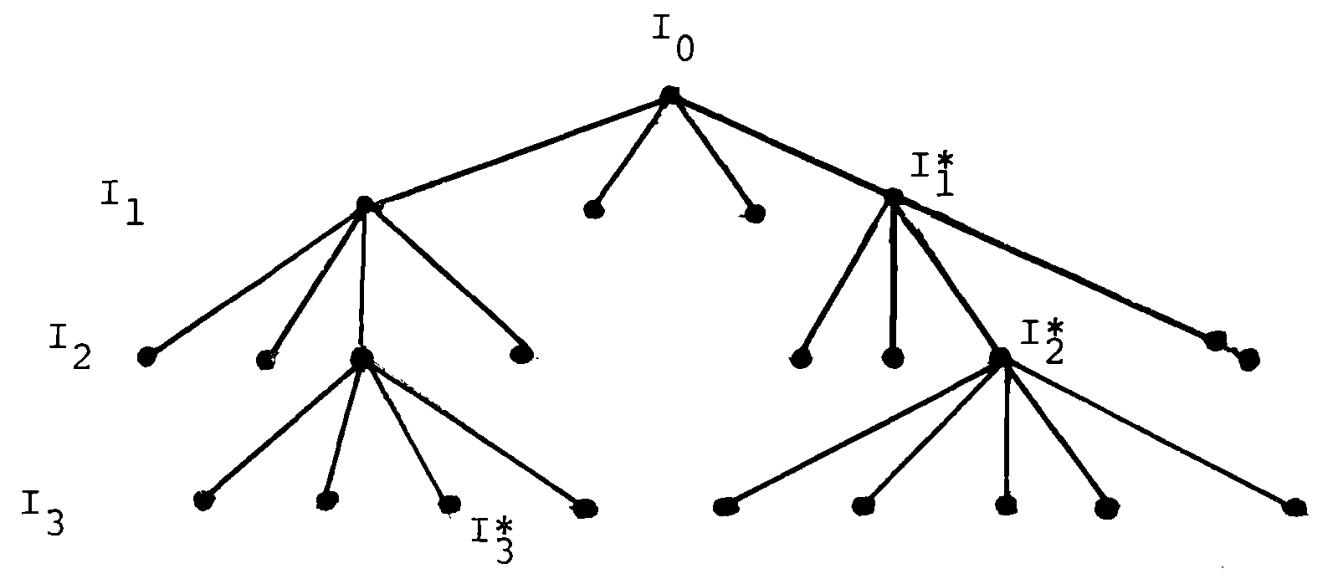

Figure 1. Branch and bound tree.

The convergence of the above branch and bound technique is guaranteed owing to the finite number of possible enumerations. The more important criterion is efficiency, an evaluation of which would require numerical computation.

IV. Optimal Control for the Backward Linkage Model

The state equations for this model are

$$
\begin{aligned}
& x(t+1)=\operatorname{Kx}(t)+(I-\tilde{M}) v(t) \\
& v(t+1)=\tilde{M} v(t)+u(t)+z(t)
\end{aligned}
$$

where $v(t)$ is the distribution of job vacancies in the $t^{\text {th }}$ time period; $u(t)$ is the distribution of government stimulated job vacancies; $z(t)$ is the distribution of spontaneously occurring vacancies; $K$ is a diagonal matrix with diagonal elements being the regional natural population growth rates; $\widetilde{M}$ is a migration matrix with elements $\tilde{\mathrm{m}}_{j k}$, the probability that a job vacancy in region $\mathrm{k}$ will be filled by someone living in $j$. (See Evtushenko and Mackinnon [4].)

The control variables in this model are $u(t)$ which are subject to budgetary and nonnegativity constraints of the same type as Eq. (2) to (4). The performance index $J$ is of the same form as Eq. (5).

The above problem is more general than the one considered earlier in section II in the sense that the dimension of the control vector $u(t)$ is smaller than the dimension of the total 
state vector viz. $(x(t), v(t))$. One approach would be to cast this problem into a general Iinear-quadratic form (3) with terminal state and in-path control variable inequality constraints. A Riccati equation approach mixed with integer programming will be used to obtain the controls in a feedback form. Clearly all the results of this section may be specialized to the forward linkage model, though extra costs in computation time will be incurred to obtain controls in feedback form.

Define a new state variable a(t) as follows:

$$
a(t+1)=a(t)+r^{T}(t) u(t), a(0)=0
$$

then

$$
a(T)=\sum_{t=0}^{T-1} r^{T}(t) u(t) \leq b .
$$

We assume that the binding or the nonbinding nature of constraint (24) has been determined by the branch and bound procedure. We consider the case

$$
\mathrm{a}(\mathrm{T})=\mathrm{b}
$$

Now we use the branch and bound technique to convert the control variable inequality constraints to equality constraints. Along any branch of the tree (Figure 1 ), the control variable constraints may be expressed as

$$
N(t) u(t)=\vec{u}(t)
$$

where

$$
N(t)=\pi, \bar{u}(t)=\hat{u}(t) \text { if } u(t)>0 \quad \text { (only } \underset{(3)}{E q} \text { holds) }
$$

and

$$
N(t)=\left[\begin{array}{c}
\pi \\
E(t)
\end{array}\right], \quad \bar{u}(t)=\left[\begin{array}{c}
\hat{u}(t) \\
0
\end{array}\right]
$$

if some of the controls are identically zero. The matrix $E(t)$ is a $k \times m$ matrix of zero and ones where $m$ is the dimension of $u(t)$ and $k$ is the number of control variables that are identically 
zero at time t. For example, if only the $j^{\text {th }}$ control variable is zero, then $E(t)$ is a $1 \times n$ vector of zeros except for a one in the $j^{\text {th }}$ column.

Define an augmented state vector

$$
y(t)=\left[\begin{array}{l}
x(t) \\
v(t) \\
a(t)
\end{array}\right]
$$

Eq. (21), (22) and (23) may be written, then, as

$$
y(t+1)=F(t) y(t)+G(t) u(t)+c(t)
$$

where

$$
\begin{aligned}
& F(t)=\left[\begin{array}{ccc}
K, & \mathrm{I}-\tilde{\mathrm{M}} & , \\
0, & \tilde{\mathrm{M}} & 0 \\
0, & 0 & 1
\end{array}\right] \\
& G(t)=\left[\begin{array}{c}
0 \\
I \\
r^{T}(t)
\end{array}\right] \\
& c(t)=\left[\begin{array}{c}
0 \\
z(t) \\
0
\end{array}\right], y(0)=\left[\begin{array}{l}
x_{0} \\
v_{0} \\
0
\end{array}\right]
\end{aligned}
$$

The terminal state constraint (25) may be expressed as

$$
\mathrm{Hy}(\mathrm{T})=\mathrm{b}
$$

where

$$
H=[0,0, \ldots, 1] .
$$


The cost function may be written as

$$
J=\frac{1}{2} \sum_{t=1}^{T}(y(t)-\tilde{g}(t))^{T} \tilde{Q}(t)(y(t)-\tilde{g}(t))
$$

where $\tilde{g}(t)$ and $\tilde{Q}$ are easily defined in terms of $g(t)$ and $Q(t)$. Notice that the terminal constraint (32) may be added to $\mathrm{J}$ using a quadratic penalty term which would only modify the last diagonal term of $\widetilde{Q}$ at time $T$. We would, however, handle this constraint directly in the same fashion as was done in Bryson and Ho [2]. We initially consider a somewhat more general cost function of the following type:

$$
\begin{aligned}
J^{\prime}= & \frac{1}{2} \sum_{t=0}^{T-1}\left\{(y(t)-\tilde{g}(t))^{T} \tilde{Q}(t)(y(t)-\tilde{g}(t))\right. \\
& \left.+u^{T}(t) R u(t)\right\}+\frac{1}{2}(y(T)-\tilde{g}(T)) T \tilde{Q}(T)(y(T)-\tilde{g}(T)),
\end{aligned}
$$

where $R \geq 0$ and $\tilde{Q}(0)=0$. The results for cost function are easily obtained by setting $R=0$ in the results given below.

The optimal control problem is defined by Eqs. (26), (28), (32) and (34). It is more general than the linear quadratic control problem owing to the introduction of Eq. (26). We generalize the procedure of Bryson and Ho [2, pp. 158-164] for solving this problem.

The Lagrangian function is defined by adjoining constraints $(26),(28)$ and $(32)$ to $J^{\prime}$ using multipliers $\xi(t), \lambda(t)$ and $\mu$.

$$
\begin{aligned}
\mathscr{L}= & \frac{1}{2}(Y(T)-\tilde{g}(T)) T \tilde{Q}(T)(Y(T)-\tilde{g}(T))+\mu(\text { Hy }(T)-b) \\
& -\lambda^{T}(0) Y(0)+\lambda^{T}(T) Y(T) \\
& +\sum_{t=0}^{T-1}\left\{\frac{1}{2}(y(t)-\tilde{g}(t))^{T} \tilde{Q}(t)(y(t)-\tilde{g}(t))+\frac{1}{2} u^{T}(t) R u(t)\right.
\end{aligned}
$$




$$
\begin{aligned}
& +\lambda^{T}(t) y(t)-\lambda^{T}(t+1)(F y(t)+G u(t)+c(t)) \\
& \left.+\xi^{T}(t)(N(t) u(t)-\bar{u}(t))\right\} .
\end{aligned}
$$

Setting $\frac{\partial \mathscr{L}}{\partial \mathrm{y}(\mathrm{t})}=0$ and $\frac{\partial \mathscr{L}}{\partial \mathrm{u}(\mathrm{t})}=0$ for all $\mathrm{y}(\mathrm{t})$ and $\mathrm{u}(\mathrm{t})$, we obtain $\lambda(t)=F^{T} \lambda(t+1)-\tilde{Q}(t)(Y(t)-\tilde{g}(t)), t=1, \ldots, T-1$

$\lambda(T)=-\tilde{Q}(T)(y(T)-\tilde{g}(T))-\mu H^{T}$

$u(t)=R^{-1}\left\{G^{T} \lambda(t+1)-N^{T}(t) \xi(t)\right\}$.

We now use the "sweep-method" (3) to solve the inhomogeneous Iinear two-point-boundary-value-problem (TPBVP) defined by Eqs. (28) to (31) and (36) to (38). Let

$$
\lambda(t)=-S(t) Y(t)+A(t) \mu+\alpha(t)
$$

and

$$
\Psi=H y(T)-b=D(t) y(t)+B(t) \mu+B(t),
$$

where dimensions of $S(t), A(t), D(t), \alpha(t), B(t)$ and $B(t)$ are easily defined. From Eq. (37), $S(T)=\tilde{Q}(T), A(T)=-H^{T}$, $\alpha(T)=\tilde{Q}(T) \tilde{g}(T), D(T)=H, B(T)=0, \beta(T)=-b$.

From Eq. (36),

$$
\begin{aligned}
-S(t) y(t) & +A(t) \mu+\alpha(t)=F^{T}[-S(t+1) y(t+1) \\
& +A(t+1) \mu+\alpha(t+1)]-\tilde{Q}(t)(y(t)-\tilde{g}(t)) .
\end{aligned}
$$

Substituting from Eq. (28),

$$
\begin{aligned}
-S(t) Y(t) & +A(t) \mu+\alpha(t)=-F^{T} S(t+1)[F Y(t)+G u(t)+c(t)] \\
& +F^{T}(A(t+1) \mu+\alpha(t+1))-\tilde{Q}(t)(Y(t)-\tilde{g}(t))
\end{aligned}
$$


From Eq. (38),

$$
\begin{aligned}
R u(t)= & G^{T}(-S(t+1) Y(t+1)+A(t+1) \mu+\alpha(t+1)) \\
& -N^{T}(t) \xi(t) \\
= & -G^{T} S(t+1)(F Y(t)+G u(t)+c(t))+G^{T} A(t) \mu \\
& +G^{T} \alpha(t)-N^{T}(t) \xi(t) .
\end{aligned}
$$

Thus,

$$
\begin{aligned}
u(t)= & \left(R+G^{T} S(t+1) G\right)^{-1}\left\{-G^{T} S(t+1)(F Y(t)+c(t))\right. \\
& \left.+G^{T}(A(t+1) \mu+\alpha(t+1))-N^{T}(t) \xi(t)\right\} .
\end{aligned}
$$

Setting $N(t) u(t)=0$, we can solve for $\xi(t)$ using Eq. (43)

$$
\begin{aligned}
\xi(t)= & {\left[N(t)\left(R+G^{T} S(t+1) G\right)^{-1} N^{T}(t)\right]^{-1} N(t)\left(R+G^{T} S(t+1) G\right)^{-1} } \\
& \left\{-G^{T} S(t+1)(F Y(t)+c(t))+G^{T}(A(t+1) \mu+\alpha(t+1))\right\} .
\end{aligned}
$$

Substituting back for $\xi(t)$ in Eq. (43), the folloiwng feedback control law is obtained:

$$
u(t)=L(t) y(t)+M(t) \mu+\gamma(t)
$$

where

$$
\begin{aligned}
L(t)= & -\left(R+G^{T} S(t+1) G\right)^{-1}\left\{I-N^{T}\left[N\left(R+G^{T} S G\right)^{-1} N^{T}\right]^{-1}\right. \\
& \left.N\left(R+G^{T} S G\right)^{-1}\right\} G^{T} S(t+1) F
\end{aligned}
$$




$$
\begin{aligned}
M(t)= & \left(R+G^{T} S G\right)^{-1}\left\{I-N^{T}\left[N\left(R+G^{T} S G\right)^{-1} N^{T}\right]^{-1} N\left(R+G^{T} S G\right)^{-1}\right\} \\
& G^{T} A(t+1)
\end{aligned}
$$

and

$$
\begin{aligned}
\gamma(t)= & \left(R+G^{T} S G\right)^{-1}\left\{-G^{T} S c+G^{T} \alpha-N^{T}\left[N\left(R+G^{T} S G\right)^{-1} N^{T}\right]^{-1} N\right. \\
& \left(R+G^{T} S G\right)^{-1}\left(-G^{T} S C+G^{T} \alpha\right\} .
\end{aligned}
$$

Notice that $N(t) L(t)=0, N(t) M(t)=0$ and $N(t) \gamma(t)=0$, so that $N(t) u(t)=0$ is automatically satisfied.

We now derive recursive equations for $S(t), A(t)$, and $\alpha(t)$ using Eq. (42) and matching terms on both sides. This gives

$$
\begin{aligned}
& S(t)=F^{T} S(t+1) F+F^{T} S(t+1) G L(t)+\tilde{Q}(t) \\
& A(t)=F^{T} A(t+1)-F^{T} S(t+1) G M(t) \\
& \alpha(t)=F^{T} \alpha(t+1)-F^{T} S(t+1)(\gamma(t)+c(t))+\tilde{Q}(t) g(t)
\end{aligned}
$$

Equations for $D(t), B(t)$ and $B(t)$ are derived by considering Eq. (40) at times $t$ and $(t+1)$,

$$
\begin{aligned}
D(t+1) & {[F Y(t)+G L(t) y(t)+G M(t) \mu+G Y(t)+c(t)] } \\
& +B(t+1) \mu+B(t+1)=D(t) y(t)+B(t) \mu+B(t)
\end{aligned}
$$

Comparing terms in Eq. (51),

$$
\begin{aligned}
D(t) & =D(t+1)(F+G L(t)) \\
B(t) & =B(t+1)+D(t+1) G M(t) \\
B(t+1) & =B(t)-D(t+1)(G \gamma(t)+C(t))
\end{aligned}
$$


It is easily seen that $D(t)=A^{T}(t)$, so that

$$
\begin{aligned}
& B(t)=B(t+1)+A^{T}(t+1) G\left(R+G^{T} S G\right)-1 \sum(t) G^{T} A(t+1) \\
& B(T)=0
\end{aligned}
$$

where $\sum(t)=I-N^{T}\left[N\left(R+G^{T} S G\right)^{-1} N^{T}\right]-1 N\left(R+G^{T} S G\right)^{-1}$.

Eq. (55) shows that $B(t) \geq 0$ and for a normal problem $B(0)>0$. Thus Eq. (40) may be solvéd for $\mu$ as,

$$
\mu=B^{-1}(0)\left[-A^{T}(0) y(0)-b-B(0)\right] \text {. }
$$

We now summarize the solution procedure for a specific $N(t)$ sequence. The determination of optimal $N(t)$ is done by the branch and bound method of section III.

a) Solve Eqs. (48), (49), (50), (53) and (54) backward in time from $t=T$ to $t=0$. Also compute simultaneously $L(t)$, $M(t)$ and $\gamma(t)$ and store these values for all $t$.

b) Compute $\mu$ using Eq. (56). Now solve Eq. (28) forward in time using Eq. (45) to compute $u(t)$ simultaneously. The multipliers $\lambda(t)$ can be computed from Eq. (39). The multipliers $\xi(t)$ to be used in the branch and bound procedure are computed from Eq. (44). Notice that $R$ can be set to zero without any difficulty to obtain results for the cost function J (cf. Eq. (33)).

\section{Conclusions}

In this paper, a mixed integer programming optimal control (MIPOC) approach has been outlined for solving multistage decision problems with control inequality constraints. Two specific optimization problems arising in national settlement system planning were considered. The advantage of the proposed solution as compared to penalty methods is that the constraints are met exactly and all dual or shadow-price variables are computed explicitly. These variables contain valuable information for policy questions such as marginal utilities of budgets, of total immigration levels and of nonnegativity constraints. The feedback form of the control law has the additional advantage that it is also optimal for the stochastic situation in which additive random error terms are present in the model. The MIPOC approach developed here has several novel features and its applicability to constrained optimal control problems of a more general nature will be considered in other papers. 
Acknowledgments

I would like to thank Dr. R. Mackinnon for introducing me to this problem and for valuable discussions on the solution.

\section{References}

[1] Bodewig, E. Matrix Calculus. Second edition. Amsterdam, North Holland, 1959.

[2] Bryson, A.E., and Ho, J.C. Applied Optimal Control. New York, Xerox Publishing Co., 1969.

[3] Draper, N.R., and Smith, H. Applied Regression Analysis. New York, John Wiley \& Sons, 1966.

[4] Evtushenko, Y., and Mackinnon, R.D. "Non-Linear Programming Approaches to National Settlement System Planning." IIASA RR-75-26. Laxenburg, Austria, International Institute for Applied Systems Analysis, 1975.

[5] Garfinkel, R.S., and Nemhauser, G.I. Integer Programming. New York, John Wiley \& Sons, 1972 .

[6] Mackinnon, R.D. "Controlling Migration Processes of a Markovian Type." Environment and Planning. (Forthcoming). 